\title{
Patrimonio paisajístico y seguridad humana en la zona periurbana del Distrito Federal
}

\author{
María Marlene Acosta Jiménez" \\ Pedro Joaquín Gutiérrez Yurrita**
}

\begin{abstract}
La conformación urbana se ha caracterizado por modelos de apropiación y expropiación del patrimonio natural que generan paisajes con desequilibrio económico, social, cultural y ambiental; fenómeno que conlleva la pérdida de la memoria comunitaria sobre la naturaleza, genera la devastación del capital natural y el quebranto de resiliencia del ecosistema, especialmente en áreas periurbanas. Son procesos que atentan contra la seguridad humana. El adecuado manejo del patrimonio paisajistico contribuye a mejorarla brindando orientaciones para realizar actividades que respeten los valores del paisaje con base en la gestión participativa, adaptativa y el modelo administrativo de gobernanza.
\end{abstract}

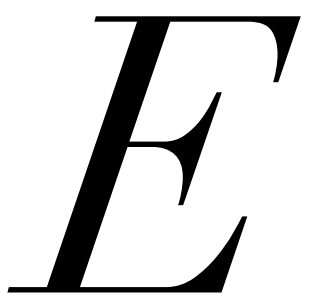

L CRECIMIENTO de las ciudades genera problemas vinculados con la movilidad, la vivienda, la contaminación ambiental, el cambio en el uso del suelo y el cambio de residencia de personas hacia los alrededores de las zonas metropolitanas, dando pie al fenómeno de la periurbanización ${ }^{1}$ y el envejecimiento del centro urba-

* Alumna del Doctorado en Ciencias en Conservación del Patrimonio Paisajístico del Centro Interdisciplinario de Investigaciones y Estudios sobre el Medio Ambiente y Desarrollo del Instituto Politécnico Nacional, México.

** Profesor del Centro Interdisciplinario de Investigaciones y Estudios del Medio Ambiente y Desarrollo del Instituto Politécnico Nacional, México. no, con lo que se incrementa la superficie del paisaje construido, el consumo energético, la exportación de calor y residuos hacia parajes adjuntos y alejados, en detrimento del paisaje natural y sus teselas conectadas en red mediante procesos de conectividad y permeabilidad ecológica. ${ }^{2}$

A pesar de ello, México crece en urbanismo a un ritmo más acelerado que otros países, siendo actualmente un Estado de seres urbanos y no de gente de campo; el abandono del medio rural para engrosar los cinturones periurbanos de las metrópolis mexicanas es la constante de los últimos treinta años debido, entre otras cosas, a la falta de una política de ordenación territorial integral, en donde el paisaje construido se intercale armoniosamente con el paisaje natural o 
agropecuario. Adolece la administración pública actual de la falta de algún sistema específico e integral de protección del paisaje que vincule el medio natural con las actividades humanas para hacer frente a problemas que atentan contra la seguridad humana. ${ }^{3}$

El enfoque del manejo paisajístico del territorio permite elaborar una propuesta metodológica para el estudio de paisajes periurbanos en el marco de la ciudad-región y ayuda a incentivar la formulación de una política compartida entre los gobiernos y la sociedad, que derive en la conservación del patrimonio paisajístico. La gestión de este patrimonio en el marco de la ciudad-región contribuye a mejorar la seguridad humana, brinda orientaciones para realizar actividades socioeconómicas en congruencia con los valores del paisaje, la gestión participativa, adaptativa y el modelo administrativo de gobernanza. ${ }^{4}$ El enfoque de seguridad humana permite que la política ambiental de ordenación territorial gire en torno a la intercomunicación y realimentación de los ejes social-económico-natural y tecnológico que permitan transitar hacia la sustentabilidad de la región para realizar actividades de adaptación al cambio climático y para reducir el riesgo de catástrofe ambiental y social, ${ }^{5}$ acciones que resultan fundamentales para las zonas periurbanas.

\section{Crecimiento urbano en México}

La aproximación a las problemáticas de seguridad humana en las zonas de transición del medio natural al medio urbano, incluyendo toda la gama de verdes y grises, pasando por las regiones agrícolas que les limitan y en ocasiones circundan, pasa por definir estos espacios, máxime cuando se los asocia con áreas y grupos de población segregada con diversos grados de marginación y títulos de propiedad o de invasión. ${ }^{6}$

De igoo a ig3o la Ciudad de México comprendía las delegaciones Cuauhtémoc, Miguel Hidalgo, Venustiano Carranza y Benito Juárez. A partir de I940 inicia una expansión urbana y territorial y se intensifica la segregación entre grupos sociales: los de mayores ingresos se desplazaron hacia el sur y el occidente de la ciudad, los de escasos recursos económicos hacia el norte y el oriente. Entre I940 y 1970 tiene lugar el fenómeno de metropolización, mientras que en el interior del Distrito Federal se consolida un segundo contorno con las delegaciones Álvaro Obregón, Azcapotzalco, Coyoacán, Cuajimalpa, Gustavo A. Madero, Iztacalco e Iztapalapa. También inicia la expan- sión hacia municipios del Estado de México como Nezahualcóyotl, Naucalpan, Tlalnepantla y Ecatepec. De I970 a r99o la expansión continúa hacia delegaciones del sur y se prolonga hacia los municipios como Coacalco o Atizapán de Zaragoza (Estado de México). En la década de los ochenta inicia el proceso de megalopolización y de $199^{\circ}$ a 2000 se incorporan nuevas periferias a la zona metropolitana.?

En la segunda década del siglo XXI el Distrito Federal está inmerso en la Región Central del País (RCP) que junto con Hidalgo, Estado de México, Morelos, Puebla y Tlaxcala concentran 32.5 por ciento de la población nacional en una superficie de 87,632 $\mathrm{km}^{2}$ y el mayor producto interno bruto del país. ${ }^{8} \mathrm{En}$ la RCP emergen diferentes áreas periurbanas en las que se ha incrementado el policentrismo, las diferencias y la polarización social: a nivel macro existe una división del espacio social que guarda relación con lineamientos de organización espacial. En otro nivel, se presentan cambios específicos y diferenciados por áreas, lo que origina la separación de grupos sociales y funciones urbanas haciendo evidente la desigualdad social y territorial: los grupos con mayores recursos económicos se instalan en lugares con mejores condiciones físicas y con mayor protección, los sectores con bajo nivel adquisitivo de bienes y servicios se ubican en zonas alejadas del centro poco aptas para ser habitadas, ${ }^{9}$ como las llanuras de inundación del lago de Texcoco y las laderas pronunciadas de la Sierra de Guadalupe.

En el siglo XXI, en el marco de la metrópoli y la ciudad-región, en México tiene lugar un modelo de expansión urbana representado en términos generales por la dispersión con base en actividades económicas, el desarrollo de transporte e infraestructura urbana y la desconcentración de funciones hacia ciudades medias y pequeñas. En este contexto, la periurbanización refiere el proceso de conformación de anillos periféricos concéntricos en los que se entrelazan actividades económicas y formas de vida con una apropiación y uso del suelo específicos. Las periferias son el espacio idóneo para ser ocupadas legal o ilegalmente debido al bajo costo del suelo o a su invasión facilitada por la escasa vigilancia administrativa del gobierno, lo que da por resultado un espacio periurbano habitacional de alta densidad poblacional y deficiencia de servicios en algunos casos carencia total de servicios urbanos como agua potable y drenaje-. La periurbanización implica la movilidad poblacional diaria por los traslados entre el domicilio y espacio de trabajo. ${ }^{10}$ 


\section{Retos para la gestión del patrimonio}

PAISAJÍSTICO Y SU IMPORTANCIA

PARA LA SEGURIDAD HUMANA

La seguridad humana surge como respuesta internacional a las amenazas actuales, emergentes y globales que enfrenta la humanidad; propone la protección y el empoderamiento de personas y comunidades en siete dimensiones de seguridad: económica, alimentaria, salud, ambiental, personal, comunitaria y política. El concepto de seguridad humana establece que en el sistema global interconectado todas las partes serán vulnerables mientras existan sectores en condiciones altamente sensibles. Plantea la reducción del riesgo colectivo mediante sistemas que proporcionen a las personas elementos de supervivencia, dignidad y medios de vida. Atiende los efectos de la violencia directa y busca evitar los efectos de la violencia indirecta como la privación de elementos para atender necesidades básicas, enfermedades, desastres naturales, desplazamiento de poblaciones y explotación de diferencias culturales y étnicas."

El proceso de crecimiento y expansión urbana en México coloca a la sociedad frente a procesos que atentan contra la seguridad humana. En el ámbito periurbano, los principales riesgos se presentan en todas las dimensiones de la seguridad humana.

En seguridad laboral, los retos se refieren a la creación de empleos, a la instrumentación de políticas para la co-localización de centros laborales y lugares de residencia, así como a la creación o adaptación de sistemas de movilidad urbana eficientes. ${ }^{2}$

En la dimensión ambiental, el crecimiento y expansión urbana generan desafíos que se configuran a partir de la transformación de los usos del suelo, la apropiación de los recursos naturales, el progresivo deterioro de los ecosistemas con la consecuente pérdida de sus servicios, el uso de energía y la generación de residuos, lo que se manifiesta como un desbalance entre los paisajes naturales y los construidos. En 20I0, el Distrito Federal era una de las zonas con mayor densidad poblacional $\left(5,920 \mathrm{hab} / \mathrm{km}^{2}\right)$, además de contaminación atmosférica, sobreexplotación de mantos acuíferos,ocupación y conversión del suelo deconservación, todo ello conllevaba problemas graves y agudos de salud pública.

Para el Distrito Federal y la Zona Metropolitana del Valle de México (ZMvM), uno de los riesgos más grandes que enfrentan es el vinculado al cambio cli- mático, representado por las ondas de calor y las lluvias torrenciales, favorecidas por la pérdida de áreas boscosas debido a la expansión desordenada de la ciudad. Las zonas más vulnerables son Iztapalapa, el norte de Tláhuac, el sur de Gustavo A. Madero, Azcapotzalco, Iztacalco, Venustiano Carranza, Cuajimalpa, Tlalpan y Xochimilco; así como los municipios de Ecatepec, Tecámac, Acolman, Chiahutla, Atenco, Texcoco y Tlalnepantla-poniente.

En materia de disponibilidad de agua, Iztapalapa, Tlalpan y Gustavo A. Madero son las más afectadas por el escaso suministro de agua, aunque se encuentran entre las que reciben mayor porcentaje del recurso hídrico en relación con las otras delegaciones. Con respecto a la salud, los habitantes de las delegaciones Iztapalapa, Gustavo A. Madero y Tlalpan son los más vulnerables a la pérdida de seguridad en la salud debido a los choques de calor y a inundaciones por desbordamiento de los ríos contaminados que cruzan la ciudad. Adicionalmente, Iztapalapa y Gustavo A. Madero concentran el mayor número de pobladores dentro del Distrito Federal y un porcentaje elevado de los que tienen más carencias económicas. ${ }^{3}$

La huella ecológica del Distrito Federal ayuda a vislumbrar la complejidad del problema ambiental y su impacto en la seguridad humana; el déficit ecológico es de 702,8 33 hectáreas, lo que significa que el territorio que requieren los habitantes de esta zona para mantener sus patrones de consumo y asimilación de residuos es de 4.7 veces superior al que poseen. En materia alimentaria, el Distrito Federal se abastece principalmente de productos agropecuarios de Morelos, Veracruz, Chiapas, Guerrero, Estado de México y Michoacán. ${ }^{14}$

El Distrito Federal tiene 6I, 458 hectáreas (4I por ciento) de suelo urbano y 87,3ı hectáreas (59 por ciento) de suelo de conservación, este último altamente amenazado por la presión de cambios de uso de suelo. De r97o al 2005, el Distrito Federal perdió cobertura forestal arbórea en una superficie de 8,59o hectáreas (Io por ciento del suelo de conservación). Para el periodo 2010-2030, se prevé una pérdida de cobertura forestal promedio de 2rg hectáreas anuales, lo que ocasionará la fragmentación de la cubierta forestal y el deterioro de servicios ambientales como cosecha de agua en follaje, infiltración, mantenimiento de la biodiversidad y riqueza biológica. Pérdida que ocasiona la desaparición de grupos funcionales como aves depredadoras y organismos polinizantes y el quebranto de la conectividad del paisaje, lo que pone en peligro la 
capacidad de resiliencia de las áreas verdes y arboladas del Distrito Federal. ${ }^{5}$

El 47 por ciento del suelo del Distrito Federal está sellado, por lo que existe un desequilibrio entre áreas verdes y el número de habitantes, se pierden servicios importantes para la calidad de vida de la población como la recarga y el filtrado de agua; regulación, retención y amortiguamiento de contaminantes depositados a partir de la atmósfera y se alteran los ciclos biogeoquímicos para el reciclaje de los compuestos orgánicos. ${ }^{16}$ Por otra parte, la ZMVM es una de las zonas metropolitanas de México con mayor producción de residuos sólidos por persona por día: 1.4 kg. ${ }^{17} \mathrm{La}$ Ciudad de México genera aproximadamente 58 por ciento de las emisiones de GEI, mientras que el Estado de México 42 por ciento. ${ }^{18}$

Elemento importante para la seguridad humana es la conciencia de los habitantes del Distrito Federal y de la ZMVM sobre el riesgo y vulnerabilidad ambiental que enfrentan. La urbanización transforma las estructuras e identidades sociales, alejándolas de la experiencia cotidiana del contacto con la naturaleza. Resultados de un estudio sobre las representaciones sociales de la población de Cuauhtepec en torno a la ciudad y las áreas arboladas, muestran que los contenidos conceptuales tienen mayor representación, en ellos figura la ciudad como un lugar de progreso y desarrollo para los seres humanos, hay una caracterización física de la ciudad y los beneficios como la posibilidad de conseguir empleo o vivienda, pero dentro de estas categorías el ambiente natural no se menciona. Se puede sugerir que en las áreas urbanas hay una pérdida de memoria comunitaria sobre la naturaleza como componente de la vida humana. ${ }^{19}$

\section{Estudio y gestión del paisaje: RUTA para}

\section{LA SEgURIDAd hUMANA EN zONAS PERIURBanas}

Llegar al corazón de los problemas que tienen lugar en zonas periurbanas requiere de entramados teóricos amplios e integrales y de metodologías que ayuden a la comprensión de estos procesos, sin perder de vista su carácter de sistemas complejos y dinámicos que se encuentran vinculados a lógicas que tienen lugar en el ámbito local, el de la ciudad y el de la región. En esta ruta, será posible apreciar de manera holística las dinámicas sociales, económicas, políticas, culturales y ambientales que se conjugan en las áreas periurbanas y comprender las condiciones para la seguridad huma- na. La metodología debe ayudar a descubrir las inflexiones para impulsar cambios territoriales orientados hacia la calidad del paisaje, calidad de vida y seguridad humana. ${ }^{20}$

La propuesta metodológica para el estudio y gestión del paisaje en zonas periurbanas del Distrito Federal está integrada por cuatro etapas: 1) fortalecimiento de enfoques y sustento de apreciaciones empíricas mediante la tríada conceptual: globalización, ciudadregión y paisaje; 2) trabajo de campo utilizando un conjunto de diferentes métodos y técnicas para descubrir procesos, puntos de inflexión y actores clave para gestionar el paisaje y la seguridad humana; 3) análisis y reelaboración del vínculo teoría-trabajo de campo, y 4) propuestas de gestión y política paisajística.

El concepto de globalización permite identificar los discursos que sustentan políticas que impactan la transformación del espacio, el uso de recursos naturales, la conformación territorial y el acceso al empleo. Muestra las articulaciones globales que tienen lugar a través de los flujos de dinero y productos en zonas como las periurbanas que parecen apartadas de estos procesos.

El concepto de ciudad-región es útil para comprender la jerarquía y el papel de las áreas periurbanas dentro del centro urbano y su periferia rural, natural o agropecuaria de la cual emerge y hacia la cual se extiende. Ayuda a caracterizar el movimiento, la circulación, los flujos, la porosidad y conectividad que van de lo global a la ciudad-región, los vínculos comerciales-cadenas de suministro; servicios y funciones de los espacios. Ubica los elementos que deben ser parte de una política compartida entre los gobiernos y la ciudadanía, para generar la agenda orientada a la solución de problemas que atentan contra la seguridad humana.

El concepto de paisaje es esencial para la investigación sobre la sustentabilidad y para el análisis holístico de procesos socioeconómicos, geográficos y ecológicos. El paisaje es bajo la visión descrita en los convenios internacionales: "cualquier parte del territorio tal como la percibe la población, cuyo carácter sea el resultado de la acción y la interacción de factores naturales y/o humanos". ${ }^{21}$ En el interior del Programa de Doctorado de Conservación del Patrimonio Paisajístico del Instituto Politécnico Nacional, los profesores del programa acordaron como concepto de paisaje: "El paisaje es un territorio estructurado físicamente y organizado funcionalmente, observado y delimitado bajo diferentes puntos de vista". Puede decirse que el paisaje es un lugar de conflicto, involucra lo físico, lo estético y lo social, com- 
bina elementos naturales, culturales y tecnológicos. Se percibe a partir de las prácticas consuetudinarias, tiene un perfil histórico, refiere directamente a las personas y representa para ellas un valor de identidad que les permite relacionarse con su cultura y la colectividad. El paisaje es dinámico, cambia por la evolución de las tecnologías, muestra el proceso de continua reorganización en tiempo y espacio del territorio por la demanda económica y social.

El paisaje urbano es síntesis de la evolución de un ambiente que se integra por estados anteriores e intervenciones, ${ }^{22}$ es marco en el que se constituyen las ciudades que actúan como lugares centrales de servicios y crecimiento económico. Al ser los paisajes espacios en donde se expresan las funciones ecosociales y sus procesos de cambio, mediadas por acciones económicas, ambientales, políticas, culturales y tecnológicas, también resultan en esencia, el lugar en donde se encuentran las claves para, desde lo local, hacer propuestas para la gestión y conservación del patrimonio paisajístico como un sistema compuesto por muchas localías.

Es primordial revisar la historia de cada paisaje, analizar su proceso de cambio en el marco de la ciudad-región, conocer la evolución y organización social como respuesta a un sistema económico, así como descubrir los procesos de pensamiento y sentido común que conduzcan a reencontrar el conocimiento ambiental compatible con la conservación del patrimonio paisajístico. Con la globalización, la conexión directa con la naturaleza está desapareciendo del contexto de vida cotidiana de los seres humanos..$^{23}$ Los estudios de lugar ayudan al análisis de los elementos referidos a los procesos de transformación del paisaje y sus vinculaciones con la ciudad-región; ayudan a descubrir los impactos de la globalización en los territorios y la vida de las personas.

Estos estudios no se pueden realizar sólo desde la teoría, requieren métodos de práctica. Una aproximación inicial al paisaje mediante la observación, el diario y la memoria visual de campo permiten la lectura preliminar, la formulación de las ideas para delimitar zonas de intervención, para perfilar el paisaje y sus actores clave. Después, el diagnóstico del paisaje y el estudio de su jerarquía y papel dentro de la ciudadregión se conjugan con el estudio de su historia. La fase cierra con una evaluación multicriterio para reunir a diferentes actores y propiciar la reflexión e intercambio de conocimiento para crear propuestas de políticas de gestión paisajística. El análisis hermenéutico de la norma y talleres lúdico-reflexivos ayudan a sus- tentar estas propuestas para orientarlas hacia esquemas de gobernanza democrática.

\section{Lectura del paisaje periurbano de Cunuhtepec en el marco de la Región Centro de México}

Cuauhtepec pertenece a la delegación Gustavo A. Madero, es uno de los nueve pueblos originarios en esta demarcación política, está situado a las faldas de la Sierra de Guadalupe, área natural protegida que se integró con patrimonio de los ejidos Cuauhtepec. ${ }^{24}$ Tiene una superficie de $22.22 \mathrm{~km}^{2}$ (véase mapa i) y un aproximado de 56 colonias que lo integran. Colinda con el Estado de México, cuenta con un corredor urbano de baja intensidad, caracterizado por centros de barrio con estructura lineal. Está formado por núcleos de vivienda de tipo popular y precario. Su principal equipamiento está compuesto por los deportivos Carmen Serdán y Juventino Rosas, el Reclusorio Norte, la Universidad Autónoma de la Ciudad de México (Campus Cuauhtepec), tres casas de cultura, el Hospital Materno Infantil Cuauhtepec y el Centro de Salud que dependen de la Secretaría de Salud del Distrito Federal.

En r9ı 8 Venustiano Carranza entregó la dotación para conformar el ejido de Cuauhtepec. La última ampliación fue en 1945. Paradójicamente, a partir de los años cuarenta del siglo $\mathrm{xx}$ se realizaron diversas expropiaciones a favor de organismos locales y federales, así, el ejido empezó a desaparecer. En su etapa rural Cuauhtepec era considerado zona turística por su fiesta patronal, celebraciones tradicionales y actividades de cacería. En ıяıo contaba con aproximadamente ı,ooo habitantes. ${ }^{25}$ Para 2000, la población aproximada era de 220,5io personas, lo que da una idea de la presión sobre el espacio y los elementos naturales de esta zona, en la que están registrados II asentamientos humanos irregulares de los 27 que existen en Gustavo A. Madero. ${ }^{26}$

En Cuauhtepec el mobiliario urbano es prácticamente inexistente, no hay paradas de transporte público ni contenedores para basura, la señalética existe de manera precaria; carece en muchos sitios de área peatonal en la calle (banqueta), predominan las cabinas telefónicas de compañías privadas. La traza de calles y forma de construcción de viviendas es repetitiva y caótica: indicador de la ausencia de una intervención urbana planificada y de que el incentivo urbano en este espacio fue el de tener una vivienda en propiedad. 


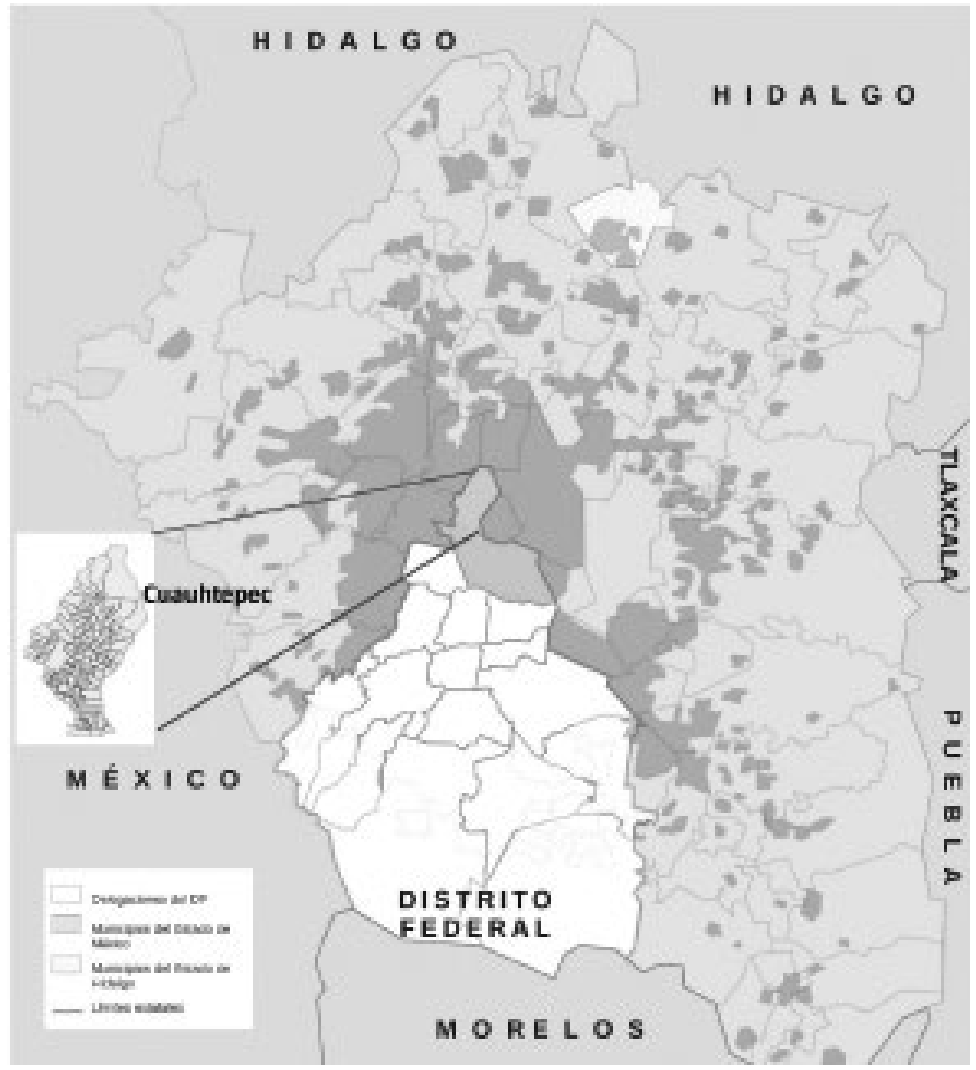

Fuente: Elaboración propia.

En las casas hay plantas, pero su distribución y el espacio que ocupan sugiere que son consideradas más como elementos de ornato que como un recurso natural que influye en la calidad de vida de las personas, ya que prefieren un espacio llano con tierra para extender su vivienda, construida por ellos mismos, que la vegetación natural del solar que invaden o compran. No hay, como se ha dicho, diseño de barrio ni de vivienda, edifican de acuerdo con sus posibilidades económicas y como va creciendo su familia. La traza urbana y la construcción indican el uso casi total del espacio para la vivienda y otros inmuebles destinados a servicios como la educación y el comercio, lo que constituye una imagen de "avasallamiento" de lo natural, para dejarla confinada a la parte alta de la Sierra de Guadalupe por la barda construida para impedir más asentamientos humanos irregulares.

En este pueblo se presenta una vivienda que se edifica de dos plantas o con un cuarto trasero para que el espacio de entrada o la planta baja sirvan para el comercio. El uso comercial en la planta baja, combinado con el uso habitacional en la parte alta quita espacio familiar; la familia no puede realizar actividades privadas por tener la puerta abierta para que entren los clientes; se pierde cohesión y sentimiento de familia. La familia tiene que hacinarse en el resto de la casa para tener espacio para los clientes y los artículos por comerciar. Cuauhtepec parece estar dividido en dos perfiles comerciales:

El de vinculación evidente con la economía del Distrito Federal. Espacio en el que se combinan negocios de autoempleo, servicios bancarios y sucursales de cadenas y empresas nacionales con presencia nacional como Elektra, Coppel y Comercial Mexicana.

De economía interna caracterizada por tianguis, comercios ambulantes y locales de autoempleo como estéticas, papelerías, tortillerías, panaderías y farmacias. Las áreas verdes y arboladas son mínimas en Cuauhtepec, el patrimonio natural más importante lo constituye el Área Natural Sierra de Guadalupe, decretada en I990 (superficie de 6’874,I94.58 hectáreas); a pesar del deterioro ambiental que sufre cuenta con alta diversidad en vegetación y fauna. La vegetación predominante es el Matorral Xerófilo y bosque de encino-pino (8o por 


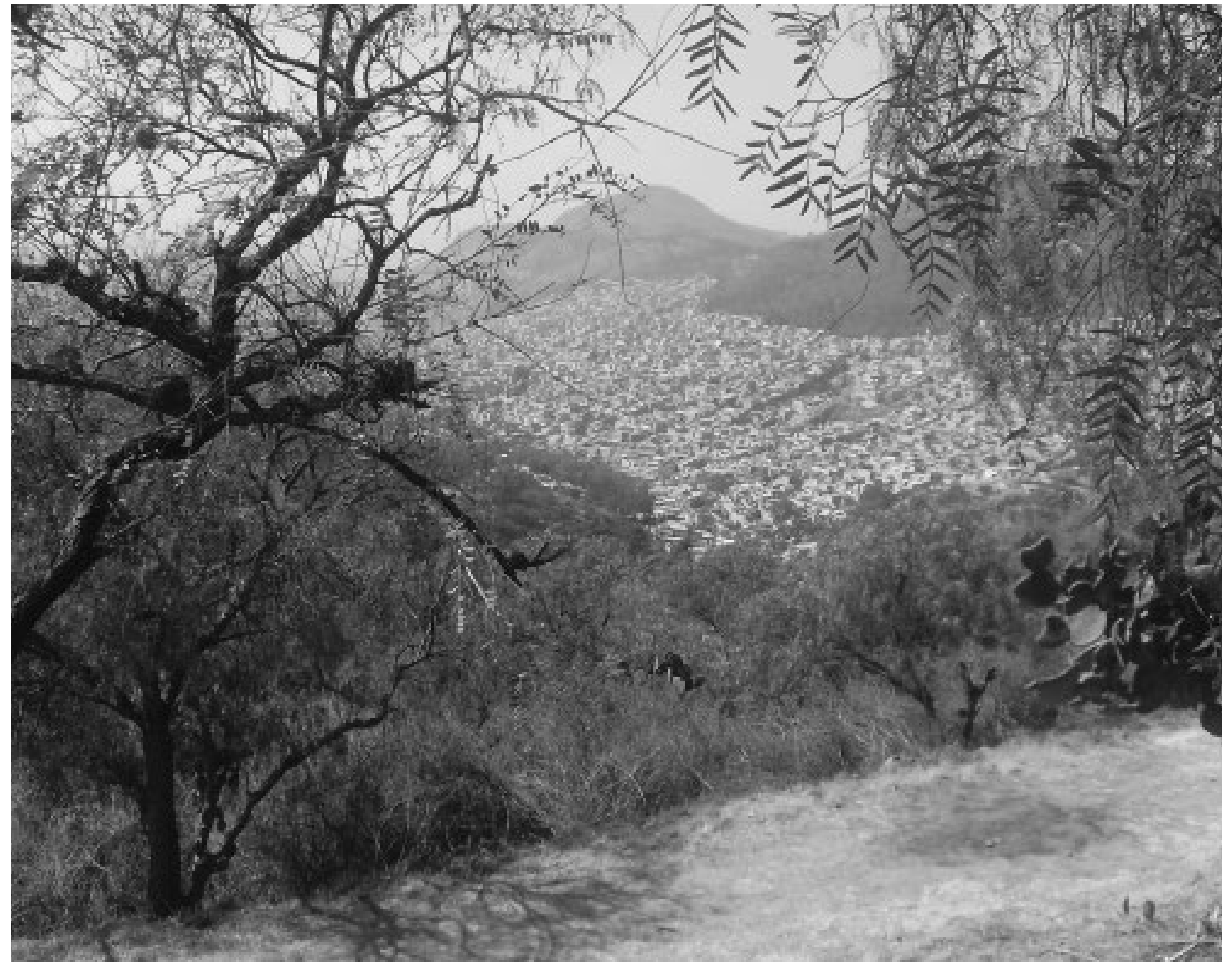

Cuauhtepec visto desde la Sierra de Guadalupe. Fotografia: Marlene Acosta.

ciento de la superficie). Se tienen registradas 3ı9 especies de plantas y 135 especies de vertebrados. ${ }^{27}$

\section{Reflexiones finales}

Al ser el paisaje un concepto que incluye diversas dimensiones que dan cuenta de procesos económicos, sociales, ambientales y tecnológicos, el trabajo de investigación para gestionar y conservar el patrimonio paisajístico requiere de la construcción de enfoques holísticos. Con la articulación de los conceptos de globalización, paisaje y ciudad-región, se genera la visión que va de lo global a lo local (glocal), lo que permite encontrar puntos de inflexión para la gestión y conservación del paisaje, gestionar la seguridad hu- mana y lograr la participación ciudadana de actores clave.

También se requieren métodos acordes con tal propósito: para recuperar la información del paisaje en términos cuantitativos y de características biofísicas, así como para rescatar, desde el enfoque cualitativo, la percepción de los actores clave para impulsar la participación ciudadana en las acciones y políticas para la seguridad humana. Este es un camino que aún requiere de bastante labor científica, aquí sólo se han perfilado ideas para impulsar este proceso. La adaptación de los programas de manejo de los paisajes y sus teselas interconectadas en las políticas públicas mediante la participación ciudadana en la toma de decisiones es fundamental para el camino a la sustentabilidad y gobernanza. 


\section{- notas $\cdot-$}

' Jesús Arroyo e Isabel Corvera (20II), “Gobernanza medioambiental en la zona metropolitana de Guadalajara: una comparación con las de Monterrey y Puebla-Tlaxcala”, en Adrián Guillermo Aguilar e Irma Escamilla (coords.), Periurbanización y sustentabilidad en grandes ciudades, México, Cámara de Diputados-LXI Legislatura-Universidad Nacional Autónoma de México-Miguel Ángel Porrúa.

2. Pedro Gutiérrez (2013), "Hacia el diseño de una ciudad sustentable", Pragma, espacio y comunicación visual, vol. 4, núm. 9, pp. 2-18.

${ }^{3}$ Alexandra Aguilar (2006), "Algunas consideraciones teóricas en torno al paisaje como ámbito de intervención institucional", Gaceta Ecológica, núm. 79, pp. 5-20.

${ }^{4}$ Pedro Gutiérrez (2011), "La gestión y el manejo holístico de la naturaleza en la era de la Tercera Cultura", en Enrique Campuzano y María de la Luz Valderrábano (comp.), Ambiente, sociedad y políticas ambientales en el México contemporáneo: Una revisión interdisciplinaria, México, Porrúa-Instituto Politécnico Nacional.

${ }^{5}$ Brenda Bravo y Pedro Gutiérrez (20I4), "Introducing a New Logical Model Based on the Holistic Approach to Risk Assessment for Environmental Disaster", Environment, Energy and Biotechnology, vol. III, núm. 73, pp. 6o-64.

${ }^{6}$ Pedro Gutiérrez, Andrea Ortega, Adriana Álvarez, Luz García y Minerva Rebollar (2014), "The Holistic Management of the Landscape of Ethnic Communities will Reduce Climate Change and Promote its Sustainability", International Journal of Environmental Science and Development, vol. 5, núm. 3, pp. 3ı7323 .

7 Margarita Pérez (20ı0), "La condición global de la Ciudad de México", en Miriam Alfie, Iván Azuara, Carmen Bueno, Margarita Pérez y Sergio Tamayo (coords.), Sistema mundial y nuevas geografías, México, Universidad Autónoma Metropolitana-Universidad Iberoamericana; y Adolfo Sánchez (2012), Informe del estado de desarrollo social en el Distrito Federal. La evolución de la Ciudad de México. Factores para el desarrollo social, México, Consejo de Evaluación del Desarrollo Social del Distrito Federal.

${ }^{8}$ Gobierno del Distrito Federal (2013), "Programa General de Desarrollo del Distrito Federal 2013-2018". Gaceta Oficial del Distrito Federal, Decimoséptima Época, I2 de septiembre, núm. ı689, tomo II.

9 Rosa Rubalcava y Martha Schteingart (2012), Ciudades divididas. Desigualdad y segregación social en México, México, El Colegio de México.

${ }^{10}$ Héctor Ávila (2009), "Periurbanización y espacios rurales en la periferia de las ciudades", Estudios Agrarios, núm. 4I, pp. 93-123.

"Francisco Rojas y Andrea Álvarez (2012), "Seguridad humana. Un estado del arte”, en Francisco Rojas (ed.), Seguridad humana: Nuevos enfoques, Costa Rica, Facultad Latinoamericana de Ciencias Sociales.

12 José Casado (2012), "La estructura policéntrica de los mercados laborales locales de la Zona Metropolitana del Valle de México", Investigaciones Geográficas, núm. 79, pp. 97-II8.

${ }^{13}$ Víctor Magaña (2012), Informe final. Estudio para sistematizar una propuesta metodológica del análisis de la vulnerabilidad actual y bajo cambio climático, México, Instituto de Geografía-
Universidad Nacional Autónoma de México-Instituto Nacional de Ecología; y Armando Sánchez, Carlos Gay y Francisco Estrada (20II), "Cambio climático y pobreza en el Distrito Federal", Investigación Económica, vol. LXX, núm. 278, pp. 45-74.

${ }^{14}$ Sergio Martínez (2012), "La huella ecológica del sistema económico y urbano actual: el caso de China y del Distrito Federal", Estudios Agrarios, núm. 41, pp.I73-I97.

${ }^{15}$ Ian Thompson (20II), "Biodiversidad, umbrales ecosistémicos, resiliencia y degradación forestal”, Unasylva, vol. 62, núm. 238, pp. 25-3o.

${ }^{16}$ Cram Silke, Helena Cotler, Luis Miguel Morales, Irene Sommer y Estela Carmona (2008), "Identificación de los servicios ambientales potenciales de los suelos en el paisaje urbano del Distrito Federal", Investigaciones Geográficas, núm. 66, pp. 97- 118 .

17 ONU-Habitat (2011), Estado de las Ciudades de México 2011, México, onU-Habitat -Sedesol.

${ }^{18}$ Adriana de Almeida y Salvador Herrera (20II), 10 Estrategias de movilidad para un Estado de México competitivo, seguro y sustentable: hacia una red integrada de transporte en la Zona Metropolitana del Valle de México, México, Centro de Transporte Sustentable México-Instituto de Políticas para el Transporte y el Desarrollo.

19 María Acosta (2013), De la participación ciudadana en las políticas ambientales para la sustentabilidad: un proceso en construcción, México, CIIEMAD-IPN.

2. David Serrano (2007), "Paisaje y políticas públicas", Investigaciones Geográficas, núm. 42, pp. 1o9-123.

${ }^{2 x}$ Consejo de Europa (2000), "Convenio Europeo del Paisaje", disponible en

http://www.cidce.org/pdj/Convenio\%2oPaisaje.pdf (consultado el 28 junio de 20I4).

22 Amador Ferrer (2009), "Paisajes urbanos", en Jaume Busquets y Albert Cortina (coord.), Gestión del paisaje, Barcelona, Ariel Patrimonio.

${ }_{23}$ Mihaela Hărmănescu y Andreea Popa (2013), "A New Landscape Perspective - Human Exercises Through Time in Environmental Perception", Procedia - Social and Behavioral Sciences, núm. 92, pp. 385-389.

${ }^{24}$ Gobierno del Distrito Federal (2010), "Programa Delegacional de Desarrollo Urbano para la Delegación Gustavo A. Madero del Distrito Federal 20го", Gaceta Oficial del Distrito Federal, Decimoséptima Época, I2 de agosto, núm. 9o3, tomo II.

${ }^{25}$ Programa de Apoyo a Pueblos Originarios (2009), Cuauhtepec: memorias de ayer y hoy, México, Secretaría de Desarrollo Rural y Equidad para las Comunidades, Gobierno del Distrito Federal.

${ }^{26}$ Procuraduría Ambiental y del Ordenamiento Territorial (20I0), Estudio sobre el ordenamiento, control y tratamiento integral de los asentamientos humanos irregulares, ubicados en suelo de conservación del Distrito Federal, México, Procuraduría Ambiental y del Ordenamiento Territorial del Distrito Federal.

27 Secretaría del Medio Ambiente (2003), "Acuerdo por el que se aprueba el Programa de Manejo del área Natural Protegida con la Categoría de Zona Sujeta a Conservación Ecológica Denominada Sierra de Guadalupe", Gaceta Oficial del Distrito Federal, Decimotercera Época, 2 de diciembre, núm. 94. 